\title{
Axion-photon conversion caused by dielectric interfaces: quantum field calculation
}

\author{
Ara N. loannisian, ${ }^{a, b}$ Narine Kazarian, ${ }^{b}$ Alexander J. Millar ${ }^{c}$ and \\ Georg G. Raffelt ${ }^{c}$
}

${ }^{a}$ Yerevan Physics Institute, Alikhanian Br. 2, 375036 Yerevan, Armenia

${ }^{b}$ Institute for Theoretical Physics and Modeling, 375036 Yerevan, Armenia

${ }^{c}$ Max-Planck-Institut für Physik (Werner-Heisenberg-Institut), Föhringer Ring 6, 80805 München, Germany

E-mail: ara.ioannisyan@cern.ch,narinkaz@gmail.com,millar@mpp.mpg.de, raffelt@mpp.mpg.de

\begin{abstract}
Axion-photon conversion at dielectric interfaces, immersed in a near-homogeneous magnetic field, is the basis for the dielectric haloscope method to search for axion dark matter. In analogy to transition radiation, this process is possible because the photon wave function is modified by the dielectric layers ("Garibian wave function") and is no longer an eigenstate of momentum. A conventional first-order perturbative calculation of the transition probability between a quantized axion state and these distorted photon states provides the microwave production rate. It agrees with previous results based on solving the classical Maxwell equations for the combined system of axions and electromagnetic fields. We argue that in general the average photon production rate is given by our result, independently of the detailed quantum state of the axion field. Moreover, our result provides a new perspective on axion-photon conversion in dielectric haloscopes because the rate is based on an overlap integral between unperturbed axion and photon wave functions, in analogy to the usual treatment of microwave-cavity haloscopes.
\end{abstract}




\section{Contents}

1 Introduction $\quad 2$

2 General transition rate $\quad 4$

2.1 First-order perturbative transition probability 4

2.2 Primakoff transition 5

2.3 Helioscope 6

3 Dielectric haloscope $\quad 7$

$\begin{array}{lll}3.1 & \text { Haloscope setup } & 7\end{array}$

3.2 Garibian wave functions 8

$\begin{array}{llr}3.3 & \text { Photon production rate } & 9\end{array}$

$\begin{array}{llr}3.4 & \text { Perfect mirror } & 10\end{array}$

$\begin{array}{ll}3.5 & \text { Many layers } \\ 3.6 & \text { Single }\end{array}$

$\begin{array}{lll}3.6 & \text { Single disk } & 11\end{array}$

4 Quantum vs. classical calculation $\quad 12$

5 Conclusions 12

A Orthogonality of Garibian wave functions 13 


\section{Introduction}

The generic two-photon interaction of axions and axion-like particles provides the basis for many possible astrophysical implications and experimental search strategies for these elusive particles. The interaction Lagrangian is usually written in the form

$$
\mathcal{L}_{a \gamma}=g_{a \gamma} \mathbf{E} \cdot \mathbf{B} a,
$$

where $\mathbf{E}$ and $\mathbf{B}$ are the electric and magnetic fields, $a$ the axion field, and $g_{a \gamma}$ a coupling constant with dimension energy ${ }^{-1}$. One obvious consequence, the decay $a \rightarrow \gamma \gamma$, is strongly phase-space suppressed for the very low-mass axions that are usually considered. Therefore, instead one frequently considers axion-photon conversion $a \rightarrow \gamma$ in the presence of external sources of electric or magnetic fields, i.e., one of the two photons is virtual and usually static. The simplest manifestation is Primakoff conversion (figure 1), where an axion converts to a photon (or the other way round) in the Coulomb field of a charged particle [1]. This process leads to axion emission in hot stellar plasmas, notably in the Sun [2].

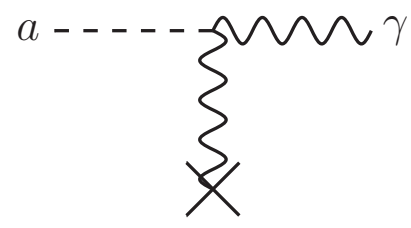

Figure 1. Primakoff conversion between axions and photons.

A somewhat different kinematical situation arises when axions or photons propagate in a macroscopic near-homogeneous magnetic field, for example solar axions propagating in the bores of the CAST dipole magnet directed toward the Sun [3]. "Primakoff scattering" is now in the forward direction and the primary axion and secondary photon remain coherent with each other. Back-conversion effects can be important, depending on detailed parameters. In this case, an intuitive description is in terms of axion-photon oscillations, analogous to neutrino flavor oscillations, here enabled by the external transverse $B$-field that mixes axions with photons [4]. This situation can also be seen as Primakoff scattering where the formation zone of the secondary photon is the entire length of the CAST magnet, or even astronomical distances in the case of photon-axion conversion in astrophysical $B$-fields.

The axion-photon oscillation picture encompasses both a description in terms of classical or quantum fields. In the presence of a strong external classical magnetic field $\mathbf{B}_{\mathrm{e}}$, the effective interaction $g_{a \gamma} \mathbf{E} \cdot \mathbf{B}_{\mathrm{e}} a$ between the photon and axion radiation fields is bilinear so that this "flavor oscillation" can be interpreted as a Bogoliubov transformation between the axion and photon creation or annihilation operators [5]. In other words, the same formalism applies if the initial beam consists of individual axions or photons or of a highly-occupied and essentially classical laser beam. The outgoing radiation inherits its quantum properties from the initial beam.

We here consider a yet different kinematical situation motivated by the usual haloscope method to search for axion dark matter [6]. The favored dark matter axion mass is broadly in the $\mu \mathrm{eV}$ range. The small galactic virial velocity of around $10^{-3} c$ thus implies a de Broglie wavelength much larger than the experimental apparatus. It consists of a strong laboratory magnet to couple the dark matter axion field with the electromagnetic (EM) field and to create microwave radiation at a detectable level. The traditional cavity experiments use a 
microwave resonator, i.e., through $\mathbf{B}_{\mathrm{e}}$ the axion field drives $\mathrm{EM}$ eigenmodes of the cavity. This process is particularly effective when the axion mass $m_{a}$ matches a cavity resonance. The traditional calculation of microwave production uses the equations of motion for the classical axion field, taken to be essentially homogeneous, and for the classical cavity mode, taken to be damped by friction and by the detector which absorbs power. A perturbative calculation provides the power output in terms of an overlap integral $g_{a \gamma} a \int d^{3} \mathbf{x} \mathbf{B}_{\mathrm{e}}(\mathbf{x}) \cdot \mathbf{E}_{\mathrm{cav}}(\mathbf{x})$, where $\mathbf{E}_{\text {cav }}(\mathbf{x})$ is the electric field configuration of the used resonant cavity mode.

A new variation of this scheme is the dielectric haloscope approach, where the conversion of dark matter axions to microwave photons is enabled by immersing one or more dielectric layers in a strong laboratory field [7-11]. The main motivation is the difficulty of building a large volume of resonant cavities at relatively large axion masses. The reference value is $m_{a}=100 \mu \mathrm{eV}$, corresponding to a microwave frequency of $25 \mathrm{GHz}$, wavelength of $12 \mathrm{~mm}$, and axion de Broglie wave length of $12 \mathrm{~m}$. The transition between dark matter axions and free microwaves is suppressed by energy-momentum conservation unless $\mathbf{B}_{\mathrm{e}}$ has a strong spatial variation to provide the required momentum transfer. In the dielectric haloscope approach, instead the photon wave function is distorted by the dielectric layers to achieve the same effect. One way to calculate the emitted microwave power is to solve Maxwell's equations, augmented with the axion terms, in the presence of dielectrics [11]. Essentially one determines the exact wave functions of the combined axion-photon system in the presence of $\mathbf{B}_{\mathrm{e}}$ and the dielectric layers, matching at every dielectric interface the axion-induced electric field with those of transmitted and reflected EM waves.

We here calculate the produced microwave power in a somewhat alternative way, motivated by the traditional treatment of transition radiation $[12,13]$. A charged particle, moving uniformly on a straight line, does not radiate due to energy-momentum conservation. However, this is different in the presence of dielectrics because free photons are now solutions of Maxwell's equations in the presence of dielectrics, i.e., they are no longer eigenstates of momentum. Likewise, for a dielectric haloscope we can perform a conventional first-order perturbative calculation for the transition between dark matter axions and these distorted photons. The result will be given in terms of a matrix element which effectively is an overlap integral similar to the traditional cavity haloscope calculation, but now involving photon "scattering states" rather than the "bound state" excitations of a cavity.

This approach gives a new perspective on the conversion of axions to photons inside a dielectric haloscope, and clarifies the relationship between the overlap integral and classical calculations. While both are mathematically equivalent, they evoke very different physical pictures. While previously developed from the classical equations through cumbersome mathematical transformations in reference [11], the overlap integral formalism emerges here very naturally from the quantum-field calculation. Further, in the classical treatment many features of the overlap integral formalism, such as the normalisation and choice of the integrated $E$ field, emerged seemingly from nowhere. In the perturbative quantum-field calculation the integrand will be obvious: one must integrate over the free Garibian photon wave functions $[14,15]$, which are uniquely determined up to time-reversals. Thus we gain a deeper understanding of the underlying physics of dielectric haloscopes. 


\section{General transition rate}

\subsection{First-order perturbative transition probability}

We consider a generic situation of a plane-wave axion interacting with a configuration of external classical static electric or magnetic fields by virtue of the interaction given in equation (1.1). We ask for the decay rate (inverse lifetime) $\Gamma_{a \rightarrow \gamma}$ of an axion to convert into a photon. This could be a plane wave with momentum $\mathbf{k}$ or another type of propagating state, notably of the type caused by the presence of dielectrics. In this case $\mathbf{k}$ represents some suitable set of quantum numbers describing the wave function. Generally we consider situations where translational invariance is broken by external agents. Therefore, the simplest approach is to use non-covariant perturbation theory in the laboratory frame. The inverse lifetime of a single quantized axion with energy $\omega_{a}$ to convert into a single photon following from elementary time-dependent perturbation theory is

$$
\Gamma_{a \rightarrow \gamma}=2 \pi \sum_{\mathbf{k}}|\mathcal{M}|^{2} \delta\left(\omega_{a}-\omega_{\mathbf{k}}\right) .
$$

Here $\mathcal{M}=\left\langle\mathrm{f}\left|H_{a \gamma}\right| \mathrm{i}\right\rangle$ is the non-covariant matrix element of the interaction Hamiltonian between the initial and final state and as such has the dimension of energy. We use natural units with $\hbar=c=1$. If $\mathcal{M}$ does not depend on $\mathbf{k}$, equation (2.1) is Fermi's Golden Rule in its simplest form $\Gamma=2 \pi|\mathcal{M}|^{2} d N / d \omega$ where $d N / d \omega$ is the density of continuum final states per unit energy.

To recall the normalisation of the fields quantized in some large but finite volume $V$ we mention that the axion field has the form

$$
\phi=\sum_{\mathbf{p}} \frac{1}{\sqrt{2 \omega_{\mathbf{p}} V}}\left(a_{\mathbf{p}} e^{-i\left(\omega_{\mathbf{p}} t-\mathbf{p} \cdot \mathbf{r}\right)}+a_{\mathbf{p}}^{\dagger} e^{i\left(\omega_{\mathbf{p}} t-\mathbf{p} \cdot \mathbf{r}\right)}\right),
$$

where $a_{\mathbf{p}}$ and $a_{\mathbf{p}}^{\dagger}$ are the usual destruction and creation operators for a quantum of momentum $\mathbf{p}$ and energy $\omega_{\mathbf{p}}=\sqrt{\mathbf{p}^{2}+m_{a}^{2}}$. (Notice that $a_{\mathbf{p}}$ and $a_{\mathbf{p}}^{\dagger}$ are dimensionless and $\phi$ had dimension energy as it should.) In this case we say that the axion plane waves have amplitude 1. A similar expression pertains to the propagating polarization components of the quantized photon field $\mathcal{A}$. For simplicity, we will often separate the normalisation from the photon wave function, writing $\mathcal{A}_{\mathbf{k}} \equiv A_{\mathbf{k}} / \sqrt{2 \omega_{\mathbf{k}} V}$, where plane-wave photons correspond to $\left|A_{\mathbf{k}}\right|=1$. However, our main interest is in photon wave functions modified by dielectrics so that $\mathbf{k}$ is a more general set of quantum numbers and $A_{\mathbf{k}}$ is a nontrivial wave function that breaks translational invariance.

As a next step we assume that the external field is given as $\mathbf{B}_{\mathrm{e}}(\mathbf{r})$ or as $\mathbf{E}_{\mathrm{e}}(\mathbf{r})$ and we consider the transition of one quantum of the axion field (2.2) to a photon with quantum numbers k. The matrix element of the interaction Hamiltonian between initial and final quantum states is then found to be

$$
\mathcal{M}=\frac{g_{a \gamma}}{2 \omega V} \int d^{3} \mathbf{r} e^{i \mathbf{p} \cdot \mathbf{r}} \mathbf{B}_{\mathrm{e}}(\mathbf{r}) \cdot \mathbf{E}_{\mathbf{k}}^{*}(\mathbf{r}) \quad \text { or } \quad \mathcal{M}=\frac{g_{a \gamma}}{2 \omega V} \int d^{3} \mathbf{r} e^{i \mathbf{p} \cdot \mathbf{r}} \mathbf{E}_{\mathrm{e}}(\mathbf{r}) \cdot \mathbf{B}_{\mathbf{k}}^{*}(\mathbf{r}),
$$

where $\omega=\omega_{a}=\omega_{\mathbf{k}}$. Moreover, $\mathbf{E}_{\mathbf{k}}(\mathbf{r})$ or $\mathbf{B}_{\mathbf{k}}(\mathbf{r})$ are the electric or magnetic field configuration associated with the unnormalised photon wave function $A_{\mathbf{k}}$. To check the dimensions of this expression, notice that the external electric or magnetic fields, in natural units, have dimension (energy $)^{2}, g_{a \gamma}$ has dimension (energy) ${ }^{-1}$, and the amplitudes $\mathbf{E}_{\mathbf{k}}$ or $\mathbf{B}_{\mathbf{k}}$ have 
dimension (energy) because $A_{\mathbf{k}}$ is dimensionless and $E_{\mathbf{k}} \sim \omega A_{\mathbf{k}}$. Overall $\mathcal{M}$ therefore has dimension (energy) as it should.

The matrix element equation (2.3) is an overlap integral of the external EM field, sandwiched between the spatial axion and photon wave functions. In the haloscope context, the axion momentum will be taken to be vanishingly small. In this sense, the microwave production rate is proportional to an overlap integral of the external EM field configuration with the photon wave function.

\section{$2.2 \quad$ Primakoff transition}

To connect to a familiar case, we consider Primakoff transition of axions to photons in the Coulomb field of a charge $Z e$. It represents a heavy nucleus which does not recoil, so its electric field $\mathbf{E}_{\mathrm{e}}(\mathbf{r})=Z e \mathbf{r} / r^{3}$ plays the role of an external static field. The photons are taken to be plane waves with wave vector $\mathbf{k}$ so that the final-state photon's magnetic field is $\mathbf{B}_{\mathbf{k}}(\mathbf{r})=i(\mathbf{k} \times \boldsymbol{\epsilon}) e^{i \mathbf{k} \cdot \mathbf{r}}$, where $\boldsymbol{\epsilon}$ is a polarization vector. Therefore, the matrix element is

$$
\mathcal{M}=i \frac{g_{a \gamma} Z e}{2 \omega V}(\mathbf{k} \times \boldsymbol{\epsilon}) \cdot \int d^{3} \mathbf{r} \frac{\mathbf{r}}{r^{3}} e^{-i \mathbf{q} \cdot \mathbf{r}},
$$

where $\mathbf{q}=\mathbf{k}-\mathbf{p}$ and the integral is $-i \mathbf{q} / \mathbf{q}^{2}$. Because $(\mathbf{k} \times \boldsymbol{\epsilon}) \cdot \mathbf{k}=0$ we find $(\mathbf{k} \times \boldsymbol{\epsilon}) \cdot \mathbf{q}=$ $(\mathbf{k} \times \boldsymbol{\epsilon}) \cdot \mathbf{p}=(\mathbf{p} \times \mathbf{k}) \cdot \boldsymbol{\epsilon}$, i.e.,

$$
\mathcal{M}=\frac{g_{a \gamma} Z e}{2 \omega V} \frac{(\mathbf{p} \times \mathbf{k}) \cdot \boldsymbol{\epsilon}}{|\mathbf{p}-\mathbf{k}|^{2}} .
$$

The final-state photons could have any polarization, so in the squared matrix element we must perform a spin sum and find

$$
\sum_{\epsilon}|\mathcal{M}|^{2}=\left(\frac{g_{a \gamma} Z e}{2 \omega V}\right)^{2} \frac{|\mathbf{p} \times \mathbf{k}|^{2}}{|\mathbf{p}-\mathbf{k}|^{4}} .
$$

Notice that in $\sum_{\epsilon}(\mathbf{p} \times \mathbf{k})_{i} \boldsymbol{\epsilon}_{i}(\mathbf{p} \times \mathbf{k})_{j} \boldsymbol{\epsilon}_{j}$ we can use $\sum_{\boldsymbol{\epsilon}} \boldsymbol{\epsilon}_{i} \boldsymbol{\epsilon}_{j}=\delta_{i j}$ because the longitudinal part of $\boldsymbol{\epsilon}$ vanishes in $(\mathbf{p} \times \mathbf{k}) \cdot \boldsymbol{\epsilon}$.

For the sum over final-state photons we instead integrate using the density of states $V /(2 \pi)^{3}$ in momentum space and include energy conservation, i.e., we use $\sum_{\mathbf{k}} \delta\left(\omega_{a}-\omega_{\mathbf{k}}\right) \rightarrow$ $V \omega^{2} \int d \Omega_{\mathbf{k}} /(2 \pi)^{3}$ with $\omega=\omega_{\mathbf{k}}=|\mathbf{k}|$. Overall we thus find

$$
\Gamma_{a \rightarrow \gamma}=\frac{1}{V}\left(\frac{g_{a \gamma} Z e}{4 \pi}\right)^{2} \int d \Omega_{\mathbf{k}} \frac{|\mathbf{p} \times \mathbf{k}|^{2}}{|\mathbf{p}-\mathbf{k}|^{4}},
$$

in agreement with the literature [16]. For massless axions the rate diverges in the forward direction, although this effect would moderated in a stellar plasma by screening effects. For axions at rest $(\mathbf{p}=0)$ the rate vanishes because the problem is now spherically symmetric and there is no induced oscillating electric or magnetic multipole that could radiate a photon.

The conversion rate on charged particles would be relevant in a stellar plasma where the relevant quantity is our result times the number of nuclei, i.e., instead of $1 / V$ it is proportional to the number density of nuclei. In other words, the factor $1 / V$ represents one charged target particle in our normalisation volume. 


\subsection{Helioscope}

As another example we consider the axion helioscope [6], i.e., a dipole magnet oriented toward the Sun such as the CAST experiment [3]. The external magnetic field is taken to be a constant value $\mathbf{B}_{\mathrm{e}}$, whereas the final-state photon is taken to be a plane wave so that $\mathbf{E}_{\mathbf{k}}(\mathbf{r})=i \omega_{\mathbf{k}} \boldsymbol{\epsilon} e^{i \mathbf{k} \cdot \mathbf{r}}$. Therefore, the matrix element is

$$
\mathcal{M}=i \frac{g_{a \gamma} \boldsymbol{\epsilon} \cdot \mathbf{B}_{\mathrm{e}}}{2 V} \int d^{3} \mathbf{r} e^{-i \mathbf{q} \cdot \mathbf{r}} .
$$

We assume $\mathbf{B}_{\mathrm{e}}$ to be transverse to the axion momentum $\mathbf{p}$, so only photons with polarization parallel to $\mathbf{B}_{\mathrm{e}}$ are produced. Moreover, our setup has translational invariance in the $y$ - $z$ directions, assuming the axion momentum is in the $x$-direction. Therefore, the $y$ and $z$ components of momentum are conserved and the photon momentum must also be along the $x$-direction. Our large quantization volume is $V=S L$ with $S$ some large area in the $y$-zplane and $L$ some large distance in the $x$-direction. The magnetic region itself is taken to have length $\ell$ in the $x$-direction, so with $\int d y d z=S$ overall the matrix element is

$$
\mathcal{M}=\frac{g_{a \gamma} B_{\mathrm{e}}}{2 L} \int_{-\ell / 2}^{+\ell / 2} d x e^{-i q x}=\frac{g_{a \gamma} B_{\mathrm{e}}}{2 L} \frac{2 \sin (q \ell / 2)}{q},
$$

where $q=k_{x}-p_{x}$. Note that there are two possibilities for $q$, depending on whether the photon is emitted forwards or backwards, $q_{ \pm}= \pm \omega-\sqrt{\omega^{2}-m_{a}^{2}}$, respectively. Energy conservation implies $\left|k_{x}\right|=\omega$.

To sum over final states, the symmetry of our setup dictates that only photon momenta $\mathbf{k}$ in the $x$-direction appear, i.e., $\sum_{\mathbf{k}} \delta\left(\omega_{a}-\omega_{\mathbf{k}}\right) \rightarrow(L / 2 \pi) \int d\left|k_{x}\right| \delta\left(\omega_{a}-\omega_{k_{x}}\right)=(L / 2 \pi)$ with $\left|k_{x}\right|=\omega_{k_{x}}$. We find for the axion transition rate

$$
\Gamma_{a \rightarrow \gamma}=\frac{1}{L} P_{a \rightarrow \gamma} \quad \text { where } \quad P_{a \rightarrow \gamma}=\sum_{q=q_{ \pm}}\left(g_{a \gamma} B_{\mathrm{e}} \frac{\sin (q \ell / 2)}{q}\right)^{2},
$$

where the sum is over the two cases of momentum transfer.

For solar axions, the x-ray energies are very large compared with the Fourier components of the magnetic field region, so we neglect the fast-oscillating part from $q_{-}=-\omega-\sqrt{\omega^{2}-m_{a}^{2}}$. In other words, "wrong direction" photons are hardly emitted because the $B$-field region does not end abruptly enough to provide the required large momentum transfer. On the other hand, the difference term $q_{+}=\omega-\sqrt{\omega^{2}-m_{a}^{2}}$ can be so small that $q_{+} \ell \ll 1$ and the conversion rate is then the usual expression $P_{a \rightarrow \gamma}=\left(g_{a \gamma} B_{\mathrm{e}} \ell / 2\right)^{2}$. Of course, if we neglect the fast-oscillating part, we are back to the usual picture of axion-photon oscillations in the spirit of neutrino flavor oscillations along the magnet pipe.

To interpret the normalisation of our result we notice that by construction we began with one axion in our normalisation volume $V=S L$. We can express our result as a photon flux per unit area and unit time emerging at the ends of the magnetic field region. Therefore, we divide $\Gamma_{a \rightarrow \gamma}$ by the area $S$, implying that the result is proportional to $1 / V$, the number of axions per normalisation volume. So the emerging photon flux is proportional to the axion number density $n_{a}$ as it should be.

In the limit $\mathbf{p} \rightarrow 0$ the helioscope becomes a haloscope, i.e., we can interpret the result as a photon flux from dark matter axion conversion emerging in two directions from a homogeneous magnetic field region (length $\ell$ ) which ends abruptly on both sides. The 
number density of axions is $n_{a}=\rho_{a} / m_{a}$ with $\rho_{a}$ the local dark matter axion mass density. Thus the number of microwave photons emerging from one side of the magnetic field region per unit time and unit area is

$$
\Phi_{\gamma}=\frac{\rho_{a}}{m_{a}}\left(g_{a \gamma} B_{\mathrm{e}} \frac{\sin \left(m_{a} \ell / 2\right)}{m_{a}}\right)^{2} .
$$

In contrast to the Primakoff process on a single charge, we here obtain a nonvanishing photon production rate in the limit of vanishing axion velocity. The external magnetic field defines a vector to produce dipole radiation driven by the oscillating homogeneous axion field.

\section{Dielectric haloscope}

\subsection{Haloscope setup}

Our real interest, however, is a setup where the quasi-homogeneous external magnetic field falls off adiabatically so that it does not provide any significant momentum transfer and the axion-photon transition rate vanishes with excellent approximation. However, we now introduce a system of parallel dielectric layers, oriented parallel to $\mathbf{B}_{\mathrm{e}}$. The simplest case of a dielectric disk is shown in figure 2. The dielectric disk breaks translation invariance and thus enables the transition between dark-matter axions and microwave photons.
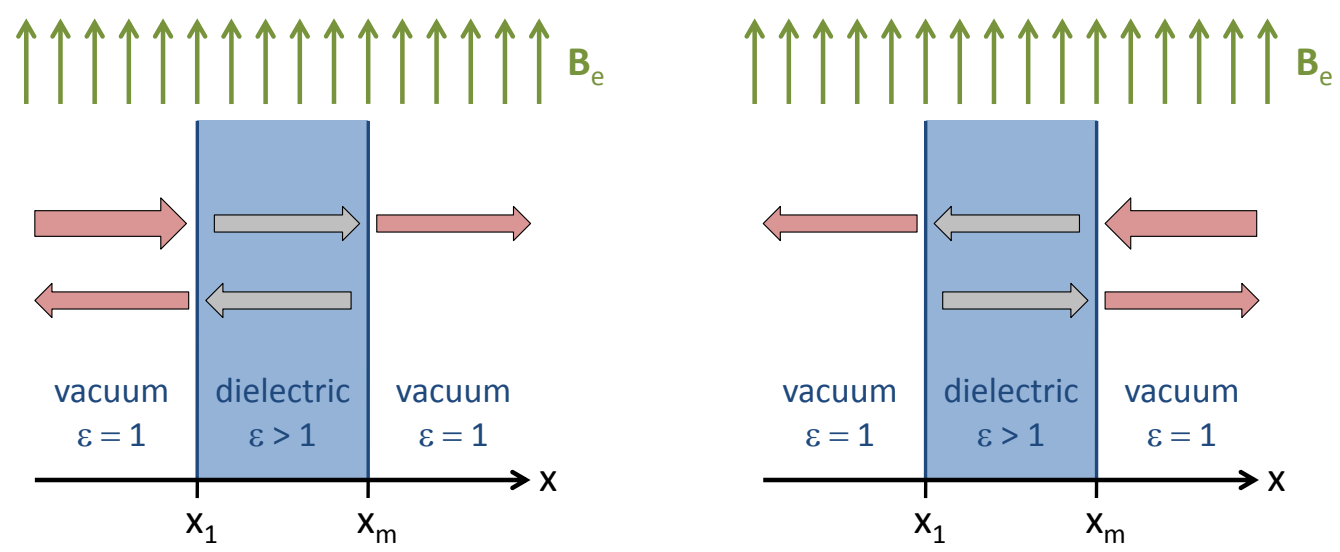

Figure 2. Dielectric haloscope. The dielectric region could be a single layer as shown or consist of many layers with the outermost interfaces at $x=x_{1}$ and $x=x_{m}$. For several layers there are many internal reflections which are not shown. The arrows indicate the photon wave functions with an incoming wave being split into a reflected and transmitted one. Reversing the arrows in both panels, there exists a second set of wave functions with two incoming waves coalescing to a single outgoing one. The shown set of wave functions or this second set are equivalent representations, as shown explicitly in reference [14].

It is now the photon wave function itself which is distorted by the dielectric layers and thus no longer an eigenstate of momentum. The same logic applies to transition radiation which arises when a charged particle traverses dielectric interfaces. While transition radiation is traditionally treated on the classical level, a quantum calculation is required to treat backreaction on the emitting particle. This point was first made by Garibian [14, 15] who defined the photon wave functions for the simple case of a dielectric interface, but the same approach applies to a dielectric disk [17] or more complicated arrangements. We will only 
consider axions with momentum p perpedicular to the interfaces, so they and the photon wave functions will be translationally invariant in the transverse directions.

\subsection{Garibian wave functions}

In this simple geometric setup, the Garibian wave functions consist of an incoming plane wave which is split by the haloscope into a transmitted and a reflected component. There are two such wave functions, depending on the side of the haloscope on which they impinge. Without the haloscope, these would be left- and right-moving plane waves with wave vector k. Equivalently, one can use the time-reversed wave functions with all momenta reversed, corresponding to two incoming waves which coalesce such as to produce only one outgoing wave on either side of the haloscope. One can use one or the other set to span the space of photon wave functions. The picture of an incoming beam being split into two outgoing waves as shown in figure 2 is somewhat more intuitive to construct the wave functions, whereas the coalescence picture is more intuitive when we ask for photon emission in one specific direction from the haloscope. Notice that in general our system is not left-right symmetric.

Explicitly we write the Garibian wave functions for the photon electric field configuration for our one-dimensional problem in the form $\mathbf{E}(\mathbf{r})=i \omega \epsilon A_{\omega}(x)$, where $\boldsymbol{\epsilon}$ is a polarization vector parallel to $\mathbf{B}_{\mathrm{e}}$. Outside of the haloscope $\left(x<x_{1}\right.$ and $\left.x>x_{m}\right)$ the $\mathrm{L}$ wave function (left panel of figure 2) has the form

$$
A_{\omega}^{L}(x)= \begin{cases}e^{i \omega \Delta x_{1}}+\mathcal{R}_{L} e^{-i \omega \Delta x_{1}} & \text { for } x<x_{1}, \\ \mathcal{T}_{L} e^{i \omega \Delta x_{m}} & \text { for } x>x_{m},\end{cases}
$$

corresponding to a plane wave with amplitude 1 impinging from the left side and $\Delta x_{j}=x-x_{j}$. Notice that we define these coefficient with reference to the left most interface $\left(x=x_{1}\right)$ for the waves on the lhs of the device and rightmost interface $\left(x=x_{m}\right)$ for those on the rhs of the device. It is reflected with the reflection coefficient $\mathcal{R}_{L}$ and transmitted with $\mathcal{T}_{L}$, where $\left|\mathcal{R}_{L}\right|^{2}+\left|\mathcal{T}_{L}\right|^{2}=1$. In general, our system is not left-right symmetric, so one needs to treat separately the case where the incoming wave impinges from the right,

$$
A_{\omega}^{R}(x)= \begin{cases}\mathcal{T}_{R} e^{-i \omega \Delta x_{1}} & \text { for } x<x_{1}, \\ e^{-i \omega \Delta x_{m}}+\mathcal{R}_{R} e^{i \omega \Delta x_{m}} & \text { for } x>x_{m} .\end{cases}
$$

In the haloscope region $x_{1}<x<x_{m}$, the wave functions can be very complicated due to the multiple reflections on many layers.

The main feature of these wave functions is that the normalisation is such that the incoming wave has amplitude 1, identical to an ordinary plane-wave photon, independently of the detailed behavior within the haloscope. This is physically clear if we think of a single photon moving toward the haloscope and if we think of it as a wave packet which does not yet know about the haloscope. It is built from ordinary plane wave components which must be the same whether or not the haloscope has been put in place. Therefore, the normalisation of its Fourier components should not be affected by the presence of the haloscope. By the same token, the Garibian wave functions for different $\omega$ and $\omega^{\prime}$ must be orthogonal. The haloscope, being a linear optical element, cannot mix photons with different frequencies.

The orthonormality of such wave functions was shown explicitly for the case of a single dielectric disk for a more complicated situation where the photons impinge at some angle [17]. This more general case was needed to treat transition radiation from a particle traversing the 
disk. Here we are considering 1D setups with many layers of dielectric material. While this situation appears more complicated, we show in Appendix A that one can use the relationship between the fields on either side of an interface to rewrite the orthonormality condition to be the same as for a free photon in a vacuum. This confirms that Garibian wave functions are the correct free photon wave functions for quantisation and use in our perturbative quantum calculation.

\subsection{Photon production rate}

To obtain the photon production rate we follow the same steps as in section 2.3 for the helioscope, except that the photon plane waves need to be replaced by Garibian wave functions. In other words, the integral expression in equation (2.9) must be substituted with

$$
\mathcal{I}_{\omega}=\int_{-\infty}^{+\infty} d x A_{\omega}(x)
$$

where we have already assumed zero-velocity axions, i.e., $\mathbf{p}=0$, implying $\omega=m_{a}$.

The integrals outside of the haloscope can be performed explicitly if we observe that the oscillating part at infinity does not contribute, so for example $\int_{x_{m}}^{\infty} d x e^{i \omega x}=i e^{i \omega x_{m}} / \omega$. Therefore the scattering amplitudes are

$$
\begin{aligned}
& \mathcal{I}_{\omega}^{L}=\frac{1-\mathcal{R}_{L}-\mathcal{T}_{L}}{i \omega}+\int_{x_{1}}^{x_{m}} d x A_{\omega}^{L}(x), \\
& \mathcal{I}_{\omega}^{R}=\frac{1-\mathcal{R}_{R}-\mathcal{T}_{R}}{-i \omega}+\int_{x_{1}}^{x_{m}} d x A_{\omega}^{R}(x) .
\end{aligned}
$$

In general, these expressions are different for $\mathrm{L}$ and $\mathrm{R}$ photons.

The photon flux per unit area and unit time emerging from the $\mathrm{L}$ or $\mathrm{R}$ side of the haloscope therefore is

$$
\Phi_{L, R}=\frac{\rho_{a}}{m_{a}}\left(\frac{g_{a \gamma} B_{\mathrm{e}}}{2}\right)^{2}\left|\mathcal{I}_{\omega}^{L, R}\right|^{2}
$$

with $\omega=m_{a}$. In particular, the photon flux emerging from the $\mathrm{L}$ side is calculated using the $\mathrm{L}$ photon as in the left panel of figure 2. Notice that we can look at this configuration as the time-reversed case with a photon emerging on the L side, using these as the appropriate "out states."

In order to connect to the previous literature we notice that in the classical treatment of Ref. [11] the photon flux was expressed in the form

$$
\Phi_{L, R}=\frac{\rho_{a}}{m_{a}}\left(\frac{g_{a \gamma} B_{\mathrm{e}}}{m_{a}}\right)^{2}\left|\mathcal{B}_{\omega}^{L, R}\right|^{2}
$$

where $\mathcal{B}_{\omega}^{L, R}$ is the left or right "boost amplitude". It expresses the electric field amplitude of the emerging EM wave in units of the electric field induced by the galactic axion field within a magnetic field region. Therefore, up to some global phase the connection to our scattering amplitude is

$$
\mathcal{B}_{\omega}^{L, R}=\frac{\omega}{2} \mathcal{I}_{\omega}^{L, R} .
$$

The boost amplitude, like the transmission and reflection coefficients, is a dimensionless quantity. 
Thus we see that from a purely QFT starting point, we can obtain the same result of the classical analysis of reference [11]. While a similar expression to equation (3.5) was derived in reference [11], it was not extended to small values of $|\mathcal{B}|$ as the integral terms at $\pm \infty$ were not handled correctly. Further, the connection between the integrand of $\mathcal{I}_{\omega}$ and the Garibian free photon wave functions was not made: classically the structure and normalisation of $\mathcal{I}_{\omega}$ could only be derived mathematically as a way to encode boundary conditions, without any physical underpinning.

\subsection{Perfect mirror}

For the simplest example, we can calculate the boost amplitude for a flat dish antenna, i.e., an interface between a mirror and vacuum [7]. In this case, there are only two regions, and, for a perfect mirror, photon modes are only supported in the vacuum region. In this case only one of $\mathcal{I}_{\omega}^{L, R}$ is non-trivial and we do not have to worry about multiple interfaces. As the $E$-field must be zero at the mirror, $\mathcal{R}=-1$. Thus we very easily get that $\mathcal{B}=-i$. As the boost amplitude is defined so that a dish antenna has $|\mathcal{B}|=1$, we find a perfect agreement with reference [11].

That both this overlap integral formalism and the transfer matrices used in reference [11] give the same result is not surprising. However, each formalism comes with very different physical interpretations. In the overlap integral formalism, the conversion of axions to photons occurs throughout the volume and axions and photons are in some sense treated separately. The free photon wave function satisfies Maxwell's equations by itself, for example canceling itself at the surface of the mirror.

The transfer matrix approach brings the interfaces to the forefront: the axion acts as a source of a discontinuity at each interface, requiring propagating EM waves to be emitted from the interface to satisfy Maxwell's equations. For a mirror, the axion induced $E$-field must cancel at the mirror with the propagating wave, giving $|\mathcal{B}|=1$. The volume only comes in via the distances between each interface, giving rise to interference effects (though power is generated throughout the device). This difference between the two pictures is highlighted in the present case of a mirror: a calculation that involves integrating over all space and one that only uses a single surface, with no volume or length scales, give the same result. Mathematically, this is explained by the fact that the volume integral can be rewritten as a sum over the surface terms coming from the boundaries of integration in each region [11].

\subsection{Many layers}

To make our result yet more explicit we assume the haloscope to consist of $m-1$ dielectric regions between $x_{1}$ and $x_{m}$, with parallel interfaces at $x_{j}$ with $j=1, \ldots, m$. (We follow the convention of reference [11] where the region left of the haloscope is region $j=0$ and the region to the right is $j=m$, here both taken to be essentially vacuum with $n_{0}=n_{m}=1$.) In each region, the refractive index is $n_{j}=\sqrt{\epsilon_{j}}$, assuming trivial magnetic permeability $\mu_{j}=1$. In each region $j$ the wave function is written in the form

$$
A_{j}(x)=\alpha_{j} e^{i n_{j} \omega \Delta x_{j}}+\beta_{j} e^{-i n_{j} \omega \Delta x_{j}},
$$

where $\alpha_{j}$ is the amplitude of the right-moving component and $\beta_{j}$ the left-moving one and we take $\Delta x_{0}=\Delta x_{1}$. We follow the same prescription as reference [11], so that the field amplitudes $\alpha_{j}$ and $\beta_{j}$ of the right and left moving EM waves are defined at the left boundary of every region, except for $\alpha_{0}$ and $\beta_{0}$ which are defined at $x_{1}$, i.e., the leftmost interface. 
Therefore, apart from a polarization vector parallel to $\mathbf{B}_{\mathrm{e}}$, the electric field of this EM wave is $E_{j}(x)=i \omega\left(\alpha_{j} e^{i n_{j} \omega \Delta x_{j}}+\beta_{j} e^{-i n_{j} \omega \Delta x_{j}}\right)$, whereas the magnetic field, orthogonal to the electric one, is $B_{j}(x)=i \omega n_{j}\left(e^{i n_{j} \omega \Delta x_{j}}-\beta_{j} e^{-i n_{j} \omega \Delta x_{j}}\right)$. At every interface, $E$ and $H$ parallel to the surface must be continuous, and because in our case $H=B / \mu=B$, these conditions are explicitly

$$
\begin{aligned}
\alpha_{j-1} e^{i \omega n_{j-1} d_{j-1}}+\beta_{j-1} e^{-i \omega n_{j-1} d_{j-1}} & =\alpha_{j}+\beta_{j}, \\
n_{j-1}\left(\alpha_{j-1} e^{i \omega n_{j-1} d_{j-1}}-\beta_{j-1} e^{-i \omega n_{j-1} d_{j-1}}\right) & =n_{j}\left(\alpha_{j}-\beta_{j}\right),
\end{aligned}
$$

where $d_{j-1}=x_{j}-x_{j-1}$ is the thickness of each dielectric layer. There are $m$ such pairs of equations for $m$ interfaces. For the case of L photons, two additional equations are $\alpha_{0}=1$ and $\beta_{m}=0$, whereas for $R$ photons we have $\alpha_{0}=0$ and $\beta_{m}=1$, so overall we have as many equations as unknown amplitudes.

Note that for $\mathrm{L}$ photons the reflection coefficient is $\mathcal{R}_{L}=\beta_{0}^{L}$ and the transmission coefficient $\mathcal{T}_{L}=\alpha_{m}^{L}$, with similar expressions for $\mathrm{R}$ waves, so the scattering amplitudes of equation (3.4) are found to be

$$
\begin{aligned}
& \mathcal{I}_{\omega}^{L}=\frac{1-\beta_{0}^{L}-\alpha_{m}^{L}}{i \omega}+\sum_{j=1}^{m-1} \frac{\alpha_{j}^{L}\left(e^{i n_{j} \omega d_{j}}-1\right)-\beta_{j}^{L}\left(e^{-i n_{j} \omega d_{j}}-1\right)}{i \omega n_{j}}, \\
& \mathcal{I}_{\omega}^{R}=\frac{1-\alpha_{m}^{R}-\beta_{0}^{R}}{i \omega}+\sum_{j=1}^{m-1} \frac{\alpha_{j}^{R}\left(e^{i n_{j} \omega d_{j}}-1\right)-\beta_{j}^{R}\left(e^{-i n_{j} \omega d_{j}}-1\right)}{i \omega n_{j}} .
\end{aligned}
$$

In general, these expressions are different for $\mathrm{L}$ and $\mathrm{R}$ photons.

\subsection{Single disk}

As a specific example we use a single dielectric disk of thickness $d$ as shown in figure 2, i.e., two interfaces at $x_{1}=-d / 2$ and $x_{2}=d / 2$, For L photons we have $\alpha_{0}=1$ and $\beta_{m}=0$ and otherwise find

$$
\begin{aligned}
& \beta_{0}=-\frac{\left(n^{2}-1\right) \sin \delta}{\left(n^{2}+1\right) \sin \delta+i 2 n \cos \delta}, \\
& \alpha_{1}=\frac{-2(n+1)}{(n+1)^{2}-(n-1)^{2} e^{i 2 \delta}}, \\
& \beta_{1}=\frac{-2(n-1) e^{i 2 \delta}}{(n+1)^{2}-(n-1)^{2} e^{i 2 \delta}}, \\
& \alpha_{2}=\frac{i 2 n}{\left(n^{2}+1\right) \sin \delta+i 2 n \cos \delta},
\end{aligned}
$$

where $\delta=n \omega d$ is the phase accrued by a photon traversing the disk. The transmission coefficient is $\mathcal{T}_{L}=\alpha_{2}$ and the reflection coefficient is $\mathcal{R}_{L}=\beta_{0}$. With these explicit results it is straightforward to evaluate the $\mathrm{L}$ scattering amplitude. We find for the corresponding boost factor

$$
\mathcal{B}_{\omega}^{L}=-i \frac{\left(n^{2}-1\right) \sin (\delta / 2)}{n^{2} \sin (\delta / 2)+i n \cos (\delta / 2)},
$$

in agreement with reference [11] up to the same overall phase of $-i$ as in the dish antenna case. 


\section{Quantum vs. classical calculation}

Thus far our calculation has assumed the transition from a single axion to a single photon, whereas galactic dark matter axions have huge occupation numbers. If we consider an axion plane wave with occupation number $N$, the axion part is of the type $|N\rangle \rightarrow|N-1\rangle$, i.e., the quantized field amplitude part of the matrix element is of the form $\langle N-1|a| N\rangle=\sqrt{N}$, where $a$ is the destruction operator. Therefore, the transition rate now picks up a factor $N$, i.e., it is now proportional to $N / V$ with $V$ the normalisation volume. In other words, we now have begun with $N$ axions instead of 1 and the photon production rate remains proportional to the axion number density.

A given momentum mode of the axion field is highly occupied, but not necessarily in a number eigenstate. The classical calculation uses a classical axion field that would be represented by a Glauber state, i.e., a superposition of number states such that it is an eigenstate of the destruction operator, not of the number operator, and the eigenvalue would be the classical field strength in analogy to an optical laser. Therefore, the photon production rate is proportional to the square of the classical axion field strength, a quantity that is the axion number density up to normalisation factors.

In general, the axion field is in some superposition of number states of the type $\phi_{\mathbf{p}}^{N}|N, \mathbf{p}\rangle$ for all momentum modes, where $\phi_{\mathbf{p}}^{N}$ is the amplitude for finding the momentum mode $\mathbf{p}$ occupied with $N$ quanta. Because the states $|N, \mathbf{p}\rangle$ are orthogonal, the overall rate is simply proportional to $\sum_{N, \mathbf{p}}\left|\phi_{\mathbf{p}}^{N}\right|^{2}$ and thus again to the total number of axions per normalisation volume.

So far we have assumed that the final-state photon state is empty before production by axions, but hypothetically it could be occupied with $N_{\gamma}$ quanta, and the rate would acquire a stimulation factor $N_{\gamma}+1$. Actually there is always an ambient bath of thermal photons unless the detector works at sufficiently cryogenic conditions. However, in this case there is also a backconversion of photons to axions which is proportional to $N_{\gamma}\left(N_{a}+1\right)$ with $N_{a}$ the axion occupation number. So the net rate is proportional to $N_{a}\left(N_{\gamma}+1\right)-\left(N_{a}+1\right) N_{\gamma}=N_{a}-N_{\gamma}$. In other words, the dominant part $N_{a} N_{\gamma}$ cancels and one cannot achieve an increased stimulated conversion rate.

The bottom line is that, as long as we are only asking for the average photon production rate, there is no difference between a classical calculation using classical axion and photon fields and a quantum-field calculation using quantized field amplitudes. A classical field has the boson property built in, so no additional coherence or stimulation factors appear. On the other hand, if one were not only to measure the average photon production rate but also its fluctuation spectrum or time correlations, then the detailed quantum properties of the axion field would feed through to the secondary EM radiation.

\section{Conclusions}

We have calculated the microwave production rate in a dielectric haloscope to search for axion dark matter. Our starting point was the ordinary perturbation theory for any quantum transition rate. The key point was to use distorted photon wave functions caused by the presence of dielectric interfaces. In contrast to Primakoff axion-photon conversion or axionphoton oscillations in large-scale magnetic fields where translational invariance is broken by the external EM field and as such by the interaction term, it is here broken by the photon wave function, regardless of the interaction term. This situation is analogous to transition 
radiation at dielectric interfaces. We have used the distorted wave functions first introduced by Garibian for a quantum calculation of transition radiation. Our final expression for the production rate involves an overlap integral between the distorted photon wave functions and the magnetic field configuration, assuming a plane-wave initial axion.

The problem of microwave production in a dielectric haloscope was previously addressed by solving the classical Maxwell equations of the coupled EM-axion system in the presence of dielectric layers. Moreover, it was shown that the final result could be transformed to an expression involving an overlap integral between certain EM solutions of Maxwell's equations without axions (see Appendix B of reference [11]). In other words, the Garibian wave functions were recovered somewhat by backward engineering through cumbersome mathematical transformations.

Our results here provide a simple and direct physical underpinning for these findings and establish a direct connection to the analogous case of transition radiation. This allows us to understand many features of the overlap integral method that were somewhat mysterious in the classical treatment: questions such as the boundaries of integration, as well as the normalisation and choice of integrands, could only be derived mathematically, with no physical underpinning. This deeper physical understanding of the overlap integral formalism is made more interesting by the fact that the transfer matrices used in the classical treatment evoke a very different physical picture. Having a clear physical understanding of both pictures gives one greater flexibility to answer questions which may be obscured in one language. Moreover, we have clarified that a classical calculation is equivalent to a traditional first-order perturbative quantum calculation so long as we only ask for the average photon production rate.

\section{Acknowledgments}

We thank Edoardo Vitagliano for helpful discussions. AI thanks the CERN theory department and the Werner Heisenberg Institute for their hospitality, where this work was done. We acknowledge partial support by the Deutsche Forschungsgemeinschaft through Grant No. EXC 153 (Excellence Cluster "Universe") and Grant No. SFB 1258 (Collaborative Research Center "Neutrinos, Dark Matter, Messengers") as well as by the European Union through Grant No. H2020-MSCA-ITN-2015/674896 (Innovative Training Network "Elusives") and Grant No. H2020-MSCA-RISE-2015/690575 (Research and Innovation Staff Exchange project "Invisibles Plus").

\section{A Orthogonality of Garibian wave functions}

To check that the Garibian wave functions used in section 3 are the correct free photon wave functions, we must check that they are eigenvalues of the Hamiltonian with the appropriate energy. In particular, for our wave functions we must confirm that [17]

$$
\frac{1}{V \sqrt{\omega \omega^{\prime}}} \int d V \frac{1}{2}\left[\epsilon \mathbf{E}^{*}\left(\mathbf{k}^{\prime}\right) \cdot \mathbf{E}(\mathbf{k})+\mathbf{B}^{*}\left(\mathbf{k}^{\prime}\right) \cdot \mathbf{B}(\mathbf{k})\right]=\omega \delta_{\mathbf{k}, \mathbf{k}^{\prime}},
$$

where for simplicity we have assumed that $\mu=1$ throughout the device, and that $\epsilon$ does not depend on $\omega$. Here $\mathbf{k}$ denotes the wavenumber of the incoming/outgoing photon wave function (i.e., the wavenumber outside the device). Recall that throughout this paper we defined $\mathbf{E}$ and $\mathbf{B}$ essentially classically in terms of the unnormalised wave function $A_{\omega}$; the factor of 
$1 / \sqrt{\omega V}$ must be restored to obtain a finite result from integrating over the Hamiltonian density. While this situation seems much more complicated then the case for plane waves, it will turn out that the contribution from every interface is exactly zero, allowing one to transform this integral into one over simple plane waves.

Consider arbitrary incoming waves of frequencies $\omega$ and $\omega^{\prime}$, specified by some $\alpha_{0}^{\prime}, \beta_{m}^{\prime}$ and $\alpha_{0}, \beta_{m}$. The primed coefficients correspond to waves of frequency $\omega^{\prime}$ and the unprimed to waves of frequency $\omega$. The integrals in the $y, z$ directions are trivial, simply pulling out the area $S$. Using Eqn. (3.8) we can write the remaining integral in a piecewise fashion,

$$
\begin{array}{r}
\frac{1}{2} \int d x\left[\epsilon E^{*}\left(\omega^{\prime}\right) E(\omega)+B^{*}\left(\omega^{\prime}\right) B(\omega)\right]=\omega \omega^{\prime} \int_{-L / 2}^{x_{1}} d x \alpha_{0}^{\prime *} \alpha_{0} e^{i \Delta x_{0} \Delta \omega}+\beta_{0}^{\prime *} \beta_{0} e^{-i \Delta x_{0} \Delta \omega} \\
+\omega \omega^{\prime} \sum_{j=1}^{m-1} n_{j}^{2} \int_{x_{j}}^{x_{j+1}} d x \alpha_{j}^{\prime *} \alpha_{j} e^{i n_{j} \Delta x_{j} \Delta \omega}+\beta_{j}^{\prime *} \beta_{j} e^{-i n_{j} \Delta x_{j} \Delta \omega} \\
+\omega \omega^{\prime} \int_{x_{m}}^{L / 2} d x \alpha_{m}^{\prime *} \alpha_{m} e^{i \Delta x_{m} \Delta \omega}+\beta_{m}^{\prime *} \beta_{m} e^{-i \Delta x_{m} \Delta \omega}
\end{array}
$$

where $\Delta \omega=\omega-\omega^{\prime}$. Fortunately, it is sufficient to consider the evaluation of the integrals at the boundary and the interior integral associated with each interface separately. Ignoring the boundary terms for now, we can rewrite the interior integral as the sum over interfaces,

$$
\frac{i \omega \omega^{\prime}}{\Delta \omega} \sum_{j=1}^{m} n_{j}\left[\alpha_{j}^{\prime *} \alpha_{j}-\beta_{j}^{\prime *} \beta_{j}\right]-n_{j-1}\left[\alpha_{j-1}^{\prime *} \alpha_{j-1} e^{i d_{j-1} n_{j-1} \Delta \omega}-\beta_{j-1}^{\prime *} \beta_{j-1} e^{-i d_{j-1} n_{j-1} \Delta \omega}\right],
$$

where we have already performed the integration. The first two terms (with a factor of $n_{j}$ ) correspond to the integral being evaluated on the rhs of the interface and the last two terms (with a factor of $n_{j-1}$ ) correspond to evaluating the integral on the lhs. To see that all terms in this sum cancel, we can look more closely at the terms coming from the lhs of the interface. From equation (3.9), we know that the $\alpha$ and $\beta$ on the lhs and rhs of an interface between media with different refractive indices are related. More specifically,

$$
\left(\begin{array}{c}
\alpha_{j} \\
\beta_{j}
\end{array}\right)=\frac{1}{2 n_{j}}\left(\begin{array}{cc}
n_{j}+n_{j-1} & n_{j}-n_{j-1} \\
n_{j}-n_{j-1} & n_{j}+n_{j-1}
\end{array}\right)\left(\begin{array}{c}
\alpha_{j-1} e^{i n_{j-1} d_{j-1} \omega} \\
\beta_{j-1} e^{-i n_{j-1} d_{j-1} \omega}
\end{array}\right) .
$$

With a little algebra, this allows us to rewrite the lhs interface terms using only quantities from the rhs of the interface,

$$
n_{j-1}\left[\alpha_{j-1}^{\prime *} \alpha_{j-1} e^{i d_{j-1} n_{j-1} \Delta \omega}-\beta_{j-1}^{\prime *} \beta_{j-1} e^{-i d_{j-1} n_{j-1} \Delta \omega}\right]=n_{j}\left[\alpha_{j}^{\prime *} \alpha_{j}-\beta_{j}^{* *} \beta_{j}\right],
$$

which makes it immediately clear that every term in equation (A.3) vanishes. Thus the only terms contributing to the integral in equation (A.2) are the boundary terms evaluated at the edge of the normalisation volume $( \pm L / 2)$. To deal with these, we can use that conservation of energy requires the incoming and outgoing waves to be related by $\mathrm{SU}(2)$ matrices, and that we can switch left and right moving waves by changing the sign of the integration limits and $x$. This allows us to rewrite the integral in equation (A.2) as an integration over simple plane waves,

$$
\omega \omega^{\prime} \int_{-L / 2}^{L / 2} d x\left[\alpha_{0}^{\prime *} \alpha_{0} e^{i x \Delta \omega}+\beta_{m}^{\prime *} \beta_{m} e^{-i x \Delta \omega}\right]=\omega^{2} L\left(\alpha_{0}^{\prime *} \alpha_{0}+\beta_{m}^{* *} \beta_{m}\right) \delta_{\omega, \omega^{\prime}}
$$


where the equality holds for very large $L$ due to the oscillatory nature of the integral. Note that our Garibian wave functions are the only solutions that have definite asymptotic values of momentum, i.e., can be associated with a single traveling photon entering/exiting the haloscope. For these waves, as either $\left|\alpha_{0}\right|=1$ or $\left|\beta_{m}\right|=1$ so equation (A.1) must hold. Thus we see that our Garibian wave functions have the correct orthonormality conditions, and so can be used to quantise the photon field for use in section 3 .

\section{References}

[1] D. A. Dicus, E. W. Kolb, V. L. Teplitz and R. V. Wagoner, Astrophysical bounds on the masses of axions and Higgs particles, Phys. Rev. D 18 (1978) 1829.

[2] G. G. Raffelt, Astrophysical axion bounds diminished by screening effects, Phys. Rev. D 33 (1986) 897.

[3] V. Anastassopoulos et al. (CAST Collaboration), New CAST limit on the axion-photon interaction, Nature Physics, in press (2017) [arXiv:1705.02290].

[4] G. Raffelt and L. Stodolsky, Mixing of the photon with low mass particles, Phys. Rev. D 37 (1988) 1237.

[5] G. Raffelt, G. Sigl and L. Stodolsky, Quantum statistics in particle mixing phenomena, Phys. Rev. D 45 (1992) 1782.

[6] P. Sikivie, Experimental tests of the invisible axion, Phys. Rev. Lett. 51 (1983) 1415; Erratum ibid. 52 (1984) 695.

[7] D. Horns, J. Jaeckel, A. Lindner, A. Lobanov, J. Redondo and A. Ringwald, Searching for WISPy cold dark matter with a dish antenna, JCAP 1304 (2013) 016 [arXiv:1212.2970].

[8] J. Jaeckel and J. Redondo, An antenna for directional detection of WISPy dark matter, JCAP 1311 (2013) 016 [arXiv:1307.7181].

[9] J. Jaeckel and J. Redondo, Resonant to broadband searches for cold dark matter consisting of weakly interacting slim particles, Phys. Rev. D 88 (2013) 115002 [arXiv:1308.1103].

[10] A. Caldwell et al. (MADMAX Working Group), Dielectric haloscopes: A new way to detect axion dark matter, Phys. Rev. Lett. 118 (2017) 091801 [arXiv:1611.05865].

[11] A. J. Millar, G. G. Raffelt, J. Redondo and F. D. Steffen, Dielectric haloscopes to search for axion dark matter: Theoretical foundations, JCAP 1701 (2017) 061 [arXiv:1612.07057].

[12] V. L. Ginzburg and V. N. Tsytovich, Several problems of the theory of transition radiation and transition scattering, Phys. Rept. 49 (1979) 1.

[13] V. L. Ginzburg and V. N. Tsytovich, Transition radiation and transition scattering, (Hilger, New York, 1990)

[14] G. M. Garibian, Phenomenological quantum electrodynamics in the case of two media, Sov. Phys. JETP 12 (1961) 1138 [J. Exptl. Theoret. Phys. (U.S.S.R.) 39 (1960) 1630].

[15] G. Garibian and C. Yang, X-ray transition radiation, Armenian Science Academy Press, 1983 (in Russian).

[16] G. G. Raffelt, Plasmon decay into low mass bosons in stars, Phys. Rev. D 37 (1988) 1356.

[17] W. Grimus and H. Neufeld, Transition radiation of ultrarelativistic neutral particles, Phys. Lett. B 344 (1995) 252 [hep-ph/9410245]. 